\title{
TYPE I DIABETES MELLITUS - DIAGNOSIS BASED ON COMPLICATIONS
}

\author{
Lorena Elena Melit, Oana Marginean, Cristina Campean, \\ Maria Oana Marginean \\ Paediatric Clinic, University of Medicine and Pharmacy Targu-Mures, Romania
}

\begin{abstract}
We present you an atypical case of type 1 diabetes mellitus at onset, with multiple abscesses, diagnosis confirmed afterwards by laboratory tests. This report is of a 14 year-old girl, who presented with abscesses of thighs, hypogastric and inguinal area. The regular investigations performed pointed out hyperglycemia with glucose in the urine, accompanied by weight loss, polyuria and polydipsia. The abscesses were incised and drained. The pathological signs of the clinical examination were: influenced general status, decreased skin turgor, dry lips, multiple fistulized abcesses of the thighs and hipogastric area, hyperemic pharynx and tonsils. The blood tests revealed hyperglycemia $(272 \mathrm{mg} / \mathrm{dl})$, leucocytosis with granulocytosis, thrombocytosis, and increased values of hemoglobin. The clinical and paraclinical data were suggestive for type I diabetes mellitus, complicated with abscesses. The treatment consisted in substitutive treatment with insulin associated with antibiotics, antimycotics, analgesics, antipyretics, endovenous perfusions for rehydration. The evolution of the case was favorable under treatment with insulin, the condition of the cutaneous abscesses improved very much after surgical treatment and administration of antibiotics, but they healed only after the equilibration of diabetes mellitus.

In conclusion, even though in the specialty literature it is reported that there appear multiple cutaneous infections in patients with diabetes mellitus wrong treated, these are rarely diagnosed at the onset of this disorder in children, this fact being probably explained by the acute onset of the disease at this age.
\end{abstract}

Keywords: diabetes mellitus, child, complications

\section{INTRODUCTION}

Type 1 diabetes mellitus is an autoimmune disease, that consists in an autoimmune specific process, in which the antibodies directed against the $\beta$ pancreatic cells, lead to selective destruction of these ones. The destruction of these cells can precede the onset of the disease even with one year. The incidence of this pathology varies very much depending on the geographic area, thus from 57.4 cases in 100,000 per year in Finland, to 0.6 cases in 100,000 per year in India (1), fact that proves a positive correlation between environmental, genetics factors and the etiology of type I diabetes. The most important genetic factor is represented by the major antigens of histocompatibility (HLA), leading in many cases to the association of type I diabe- tes with other autoimmune pathologies, such as autoimmune tiroiditis, pernicious anemia and Addison's disease, the most frequent of which being the autoimmune tiroiditis (2).

The clinical picture of type 1 diabetes mellitus is mainly represented by polyuria, polydipsia and polyphagia, but it can also appear tiredness, muscle weakness, nausea, impairment of the visual function, abdominal pain, bowel movements disorders, impairment of the peripheral nerves, with numbness in the extremities. The onset of this pathology is usually sudden, even associated with diabetic ketoacidosis. The complications of the disease are multiple and diverse, such as infections located in different parts of the body, nephropathy, neuropathy, ophthalmologic, cardiac complications, atherosclerosis. 
The treatment of type 1 diabetes mellitus consists in lifetime supplement with insulin, associated with strict monitoring of glycaemic values, appropriate education for the patient and care-givers for being capable of recognizing the symptoms of hypoglycaemia and hyperglycaemia and to also know the management of this kind of situations, and last but not least the education of the patient's compliance for preventing situations at risk and late complications of this pathology. Because of this complications, either late or immediate, type 1 diabetes is associated with higher rated of morbidity and mortality.

We chose to present this case because we want to underline an atypical onset of type 1 diabetes, the complications being the ones that led to establishing the diagnosis.

\section{CASE PRESENTATION}

A 14 year-old patient was admitted to First Pediatrics Clinic from Târgu Mures with multiple abcesses of the hipogastric and inguinal area, and with high values of the glycaemia. The onset of the disease was two weeks ago with the appearance of some boils on the thighs and the epigastric area that transformed in real abcesses. The family doctor to whom the child presented, decided after the examination to measure the glycaemia, discovering a value of $300 \mathrm{mg} / \mathrm{dl}$, reason for which, he refers the patient to our clinic for admission.

The clinical examination pointed out: influenced general status, decreased skin turgor, dry lips, multiple fistulized abcesses of the thighs and hipogastric area, hyperemic pharynx and tonsils. The laboratory tests we performed, showed: hemoglobin 16 $\mathrm{g} / \mathrm{l}$, leucocytosis $\left(18,300 / \mathrm{mm}^{3}\right)$ with severe granulocytosis $\left(15,200 / \mathrm{mm}^{3}\right)$ and also mild trombocytosis $\left(516,000 / \mathrm{mm}^{3}\right)$; VSH: $50 \mathrm{~mm} / \mathrm{h}$; PCR: 22.34 $\mathrm{mg} / \mathrm{l}$; the deviation to the left of the peripheral smear, with normocromia and normocytosis; hyperglycaemia $(272 \mathrm{mg} / \mathrm{dl})$, glycated hemoglobin $15.48 \%$, mild hypercholesterolemia (207 mg/dl); and the urine test with glycosuria, hematuria, proteinuria, leucocyturia, ketones.

Once we administered the antibiotic and insulin treatment, the values of the laboratory test, came progressively in the normal range. After admission, was also performed a surgical consult for the incision and drainage of the abcesses, cleaning and bandaging the lesions. We also sent a sample from the puss to the laboratory for bacteriological analysis, which pointed out Staphylococcus aureus MRSA. The abdominal ultrasound did not show any pathological aspects. The normal hormonal tests excluded an associated autoimmune pathology. The evolution was slowly favorable under antibiotic and insulin treatment, the patient developing also a vulvo-vaginitis.

The final diagnosis consisted in type 1 diabetes mellitus, multiple abscesses of the thighs and hypogastric area and vulvo-vaginitis.

The treatment administered during the admission was a complex one, consisting in double therapy with antibiotics with wide sptectrum, antistaphylococcal and antimycotic (Meronem, Vancomicină, Fluconazol); hypodermic substitution with insulin (Humalog $10 \mathrm{IU}$ three times a day and Lantus $10 \mathrm{IU}$ in the evening, with dosage adjusting depending on the glycaemic values), antipyretics, painkillers and rehydration perfusions. The recommendations at discharge were: life dietary appropriate for the disease, with the monitoring of the ingested amount of carbohydrates and minimum 30 minutes of physical effort a day, therapy with insulin as it follows: fast insulin three times a day (Novorapid 8-7-8 IU/day hypodermic) and slow insulin in the evening (Lantus $10 \mathrm{IU}$ ), strict monitoring of the glycaemic values and diabetological consult in a month or emergency consult if required.

Even though in the specialty literature, type 1 diabetes mellitus is associated with high rates of morbidity and mortality, the prognosis of our case on short term is a favorable one, but regarding the long term prognosis, it depends very much of the patient's education and the compliance to treatment.

\section{DISCUSSIONS}

Type I diabetes mellitus is an autoimmune pathology whose etiology remains unknown, even though its pathogenesis is influenced by genetically and environmental factors. The haplotypes involved in the development of this pathology are DRB1*03:01-DQA1*05:01 and DQB1*02:01 (DR3) (3), and a recent study proved that DR3 haplotypes with the alleles DRB3*02:02 present a higher predisposition than alleles DRB3*01:01 (4). A study from 2015 brought proves regarding the existence of a pancreatic infections with an enterovirus in patients with type 1 diabetes mellitus, suggesting the fact that this enterovirus might contribute to the progression of the disease (5). Even though type 1 diabetes mellitus is often associated with other organ specific autoimmune diseases (2), the patient presented by us did not have any other 
pathology associated to diabetes. The number of the children diagnosed with diabetes is every day higher, either due to the fact that the incidence of this pathology is increasing or to the fact that sedentary life style in the century of computerized technology and unhealthy food consumed more and more by the children (sweets, juices, fast-foods etc), lead to the early expression of the disease (6). Otherwise, according to one study, obesity is present in at least one third of the patients with type 1 diabetes mellitus, with childhood onset (7). Nevertheless, it seems than in contrast of patients with type 2 diabetes, the values of glycated albumin are not significantly correlated with the body mass index in case of patients with type 1 diabetes mellitus (8). Thus at the same time with the increase of the obesity rate in children, the differentiation of type 1 diabetes from type 2 becomes harder and harder to be made (9). The onset of type 1 diabetes is most of the times acute, even with ketoacidosis coma, but in case of our patient, the infectious complications led to establishing this diagnosis. The majority of complications in these patients, especially the microvascular ones, are due to weak control of glycaemia, fact that is often met in case of children and adolescents $(10,11)$. Otherwise, adolescent patients, immediately after dignosis are the ones that present most often a weak control of glycaemia because they refuse to monitor frequent enough their glycaemia, are not willing to keep the diet and hate the idea of making sport (12-15). There was also described a severe complication in case of a 13 year-old patient with type 1 diabetes mellitus, after an episode of ketoacidosis, meaning glycogenic hepathopaty (16). The aim of the treatment with insulin, in fact, is to diminish as much as possible both the short and long term complications determined by this disease, and in addition to that, the early introducing of an intensive therapy with insulin can prolong the period of residual secretion of the $\beta$ cells from the pancreas, thus leading to the decrease of the exogenous insulin necessary in case of afflicted patients (17).

\section{CONCLUSIONS}

The burden of type 1 diabetes mellitus is carried especially by children and adolescents, this pathology being diagnosed most frequent during the childhood. This is also the reason for the fact that the management of this disease is very difficult, and the complications determined by the weak control of glycaemic values can have a fatal impact on the quality and length of life, thus when the complications appear even from the onset of disease, is of a major importance for the patient and the family members to receive an appropriate medical education for their life to not become just a series of hypoglycaemic episoded followed by the hyperglycaemic ones.

\section{REFERENCES}

1. Patterson C.C., Dahlquist G.G., Gyürüs E., Green A., Soltész G. Incidence trends for childhood type 1 diabetes in Europe during 1989-2003 and predicted new cases 2005-20: a multicentre prospective registration study. Lancet. 2009; 373:2027-2033.

2. Kawasaki E., Gill R.G., Eisenbarth G.S. Type 1 diabetes mellitus. In: Eisenbarth GS, ed. Molecular mechanisms of endocrine and organ specific autoimmunity. Austin, Texas: R.G. Landes Company; 1999: 149-82.

3. Erlich H., VIdes A.M., Noble J. et al. HLA DR-DQ haplotypes and genotypes and type 1 diabetes risk: analysis of the Type 1 Diabetes Consortium families. Diabetes 2008; 571084-1092 10.2337/ db07-1331

4. Erlich H.A., Valdes A.M., McDevitt S.L. et al. Next generation sequencing reveals the association of DRB3*02:02 with type 1 diabetes. Diabetes 2013; 622618-2622 10.2337/db12-1387

5. Krogvold L., Edwin B., Buanes T. et al. Detection of a low-grade enteroviral infection in the inslets of Langerhans of living patients newly diagnosed with type 1 diabetes. 2014; pii: DB_141370

6. Weets I., Leeuw I.H., Du Caju M.V. et al. The incidence of type 1 diabetes in the age group 0-39 years has not increased in Antwerp (Belgium) between 1989 and 2000: evidence for earlier disease manifestation. Diabetes Care. 2002; 25:840-846.

7. Cedillo M., Libman I.M., Arena V.C. et al. Obesity, islet cell autoimmunity, and cardiovascular risk factors in youth at onset of type 1 autoimmune diabetes. J Clin Endocrinol Metab 2015; 100(1):E82-6.

8. Hirata T., Koga M., Kasayama S. et al. Glycated albumin is not significantly correlated with body mass index in patients with acute-onset type 1 diabetes. Clin Chim Acta 2015; 438:428-51.

9. Rewers M. Challenges in diagnosing type 1 diabtetes in different populations. Diabetes Metab J 2012; 36:90-7.

10. Petitti D.B., Klingensmith G.J., Bell R.A. et al. Glycemic control in youth with diabetes: the SEARCH for diabetes in Youth Study. J Pediatr 2009; 155:668-72.

11. Dabadghao P., Vidmar S., Cameron F.J. Deteriorating diabetic control through adolescence-do the origins lie in childhood? Diabet Med 2001; 18:889-94.

12. Al-Agha A., Ocheltree A., Hakeem A. Metabolic control in children and adolescents with insulin-dependent diabetes mellitus at King Abdul-Aviz university Hospital. J Clin Res Ped Endocrinol 2011; 3(4):202-7.

13. Holl R.W., Swift P.G.F., Mortensen H.B. et al. Insulin injection regimens and metabolic control in an international survey of adolescents with type 1 diabetes over 3 years: recuslts from Hvidore study group. Eur J Ped 2003; 162(1):22-9. 
14. Gong C.X., Cao B.Y., Li Y.C. Re-evaluation of management of children under age of 18 with type 1 diabetes. Chin J Diabetes 2008; 16:172-4.

15. Gong C.X., Ni G.C., Liu M. Evaluation of management of 123 cases patients with type 1 diabetes and under age of 18 years. Chin J Diabetes 2003; 4(11): 172-5.

16. Martin J., Tomlinson P. Hepatic complications in poorly controlled type 1 diabetes mellitus: a case report. N Z Med J 2014; 127(1392):96-7.
17. The Diabetes Control and Complications Trial Research Group Effect of intensive therapy on residual $\beta$-cell function in patients with type 1 diabetes in the Diabetes Control and Complications Trial. A randomized, controlled trial. Annals of Internal Med 128, 1998; 517-523 